\title{
The consolidation of object and context recognition memory involve different regions of the temporal lobe
}

\author{
Israela Balderas, ${ }^{1}$ Carlos J. Rodriguez-Ortiz, ${ }^{1}$ Paloma Salgado-Tonda, ${ }^{1}$ \\ Julio Chavez-Hurtado, ${ }^{1}$ James L. McGaugh, ${ }^{2}$ and Federico Bermudez-Rattoni ${ }^{1-3}$ \\ ${ }^{1}$ Departamento de Neurociencias, Instituto de Fisiología Celular, Universidad Nacional Autónoma de México, Apartado Postal \\ 70-253, 04510 México D.F., México; ${ }^{2}$ Department of Neurobiology and Behavior, Center for the Neurobiology of Learning \\ and Memory, University of California, Irvine, California 92697-4545, USA
}

\begin{abstract}
These experiments investigated the involvement of several temporal lobe regions in consolidation of recognition memory. Anisomycin, a protein synthesis inhibitor, was infused into the hippocampus, perirhinal cortex, insular cortex, or basolateral amygdala of rats immediately after the sample phase of object or object-in-context recognition memory training. Anisomycin infused into perirhinal or insular cortices blocked long-term (24 h), but not short-term (90 min) object recognition memory. Infusions into the hippocampus or amygdala did not impair object recognition memory. Anisomycin infused into the hippocampus blocked long-term, but not short-term object-in-context recognition memory, whereas infusions administered into the perirhinal cortex, insular cortex, or amygdala did not affect object-in-context recognition memory. These results clearly indicate that distinct regions of the temporal lobe are differentially involved in long-term object and object-in-context recognition memory. Whereas perirhinal and insular cortices are required for consolidation of familiar objects, the hippocampus is necessary for consolidation of contextual information of recognition memory. Altogether, these results suggest that temporal lobe structures are differentially involved in recognition memory consolidation.
\end{abstract}

A critical aspect of the anterograde amnesic syndrome observed in patient $\mathrm{HM}$ and other patients with medial temporal lobe damage is the loss of recognition memory (Scoville and Milner 1957). Recognition memory is the capacity to know that something has been previously experienced, either individual stimuli or whole events (Mandler 1980; Brown and Aggleton 2001). The recognition process is generally considered to be composed of at least two components, one is the judgment of familiarity of items and the other is the recollection of contextual (spatial and/or temporal) information where items were encountered (Brown and Aggleton 2001; Yonelinas et al. 2002).

Earlier studies of amnesia produced by medial temporal lobe ablations in monkeys suggested that combined lesions of the hippocampus and amygdala accounted for severe recognition memory impairment (Mishkin 1978). However, more recent findings showed that recognition impairment was not directly related to damage in those structures but, rather, to damage to the anterior and posterior portions of the perirhinal and entorhinal cortices induced by amygdala and hippocampus aspiration (Murray and Mishkin 1998).

The issue of whether the different regions of the temporal lobe contribute in the same way to familiarity and contextual information of recognition memory remains an issue of considerable controversy. Evidence from animal studies suggests that the perirhinal cortex and the hippocampus contribute differentially to these two components of recognition memory. The findings of many studies suggested that the perirhinal cortex is critically involved in discrimination of familiarity but not in contextual memory, whereas the hippocampus appears to support contextual memory but seems not to be necessary for familiarity

${ }^{3}$ Corresponding author.

E-mail fbermude@ifc.unam.mx; fax (525) 622-5607.

Article is online at http://www.learnmem.org/cgi/doi/10.1101//m.1028008. discrimination (Ennaceur et al. 1996; Ennaceur and Aggleton 1997; Bussey et al. 1999; Mumby et al. 2002, 2005; Stupien et al. 2003; Winters et al. 2004). However, as some findings suggest that the hippocampus is involved in object recognition memory as well, the role of the hippocampus in recognition memory remains highly controversial (Broadbent et al. 2004; Rossato et al. 2007; Squire et al. 2007).

Recent findings indicate that the insular cortex is also an important temporal lobe structure involved in consolidation of recognition memory. Muscarinic receptor antagonists infused into the insular cortex known to disrupt taste recognition memory also impair object recognition memory (BermudezRattoni 2004; Bermudez-Rattoni et al. 2005).

The present study investigated the specific contributions of these medial temporal lobe structures to object and object-incontext recognition memory consolidation. In order to disrupt consolidation, anisomycin was infused into perirhinal cortex, insular cortex, hippocampus, or basolateral amygdala (BLA) immediately after object or context recognition training and memory was tested $90 \mathrm{~min}$ (short-term memory) or $24 \mathrm{~h}$ (long-term memory) later.

\section{Results}

\section{Experiments for object recognition}

During the training trial (sample phase) the groups exhibited similar time exploring each of the two identical objects (Table 1). A discrimination index was calculated as the difference in time exploring the two objects, expressed as the ratio of the total time spent exploring both objects. Two-way ANOVA indicated no significant differences between groups on the training trials (sample phases) for the groups tested later for short- or long-term memory (see Table 1). 
Table 1. Recognition indexes on sample phase in object recognition task

\section{Object recognition}

(A) Sample phas (for STM)

\begin{tabular}{|c|c|c|c|c|}
\hline & Vehicle & Anisomycin & Vehicle & Anisomycin \\
\hline$\overline{\mathrm{PRH}}$ & $0.45 \pm 0.03$ & $0.47 \pm 0.01$ & $0.45 \pm 0.02$ & $0.49 \pm 0.01$ \\
\hline IC & $0.51=$ & 0.50 & $0.48 \pm 0.01$ & 0.49 \\
\hline HIP & $0.46 \pm 0.02$ & $0.47 \pm 0.01$ & $0.49 \pm 0.02$ & $0.49 \pm 0.02$ \\
\hline BLA & $0.53 \pm 0.02$ & $0.51 \pm 0.03$ & \multirow{2}{*}{\multicolumn{2}{|c|}{$\begin{array}{c}0.50 \pm 0.00 \\
F_{(7,68)}=0.11 P=0.99\end{array}$}} \\
\hline & \multicolumn{2}{|c|}{$\mathrm{F}_{(7,42)}=1.20 P=0.32$} & & \\
\hline
\end{tabular}

Recognition indexes on sample phase (two identical objects) expressed as mean \pm SEM. (A) Sample phase of STM groups. (B) Sample phase of LTM groups. Two-way ANOVA showed no differences between groups. See Results section for a detailed statistical description. For the purpose of analysis, one of the objects was indistinctly selected as "novel." Abbreviations are as in Figure 1.

As can be seen in Figure 1A, animals were exposed to two copies of a same object (sample phase), and $90 \mathrm{~min}$ (short-term memory) or $24 \mathrm{~h}$ (long-term memory) later, a different copy of one of the objects shown before was presented together with a novel object (test). Figure 1B shows the recognition index for short-term memory for the novel objects exhibited by the animals that received vehicle or anisomycin after the training trial. Animals microinfused with either vehicle solution or anisomycin showed significant preferences for the novel object on the short-term memory test. Two-way ANOVA revealed no significant differences between groups $\left(F_{(7,42)}=0.58, N S\right)$.

Interestingly, on the long-term memory test, animal's microinfused with anisomycin in either the insular or perirhinal cortex displayed impaired discrimination between familiar and novel objects. Two-way ANOVA indicated significant differences between groups $\left(F_{(7,67)}=4.00\right.$, $P<0.001)$. Fisher's post hoc test revealed that the groups infused with anisomycin into the perirhinal or insular cortices were different from the vehicle control groups $\left(P^{\prime} \mathrm{s}<0.01\right)$.

\section{Experiments for object-in-context recognition}

In order to assess contextual recognition memory, we designed a protocol where association of objects and environmental cues were the dominant features to be remembered based on the objectcontext novelty preference paradigm (Dix and Aggleton 1999). Rats were allowed to explore two different objects in context 1 (sample 1, see Fig. 2A). Twenty-four hours later, two copies of one of the objects presented the day before were presented once again but now in a different context (sample phase 2). Anisomycin injections were administered immediately after this second sample phase. On tests, rats were placed in context 2 with a copy of each of the objects presented on sample 1 (Fig. 2A). Under these conditions, rats spend more time exploring a familiar object that is presented in a novel context than they do for a familiar object that is presented in a previously presented context. This behavior is indicated by a high recognition index (see vehicleinjected groups on Fig. 2B).

During the training trial (sample phase) the groups exhibited similar time exploring each of the two objects (Table 2). Two-way ANOVA indicated no significant differences between groups in sample phase 1 for both conditions; short-term groups $\left(F_{(7,42)}=0.90, N S\right)$, or long-term groups $\left(F_{(7,59)}=0.91, N S\right)$. Similarly, there were no significant differences in sample phase 2 ; short-term groups $\left(F_{(7,42)}=1.75, N S\right)$, or long-term groups $\left(F_{(7,59)}=0.76, N S\right)$.

As can be seen in Figure 2B during retention tests at 90 min, the animals, regardless of their experimental condition, showed a preference for the familiar objects placed in novel contexts. That is, rats microinfused with either vehicle solution or anisomycin showed the expected high recognition index on the shortterm memory test. Two-way ANOVA indicated no significant differences between groups $\left(F_{(7,42)}=0.23, N S\right)$.

Interestingly, on the long-term memory probe, only animals microinfused with anisomycin in the hippocampus displayed impaired ability to recognize familiar objects in novel contexts. Two-way ANOVA indicated differences between groups $\left(F_{(7,59)}=2.39, P<0.05\right)$. Fisher's post hoc analysis revealed that the group infused with anisomycin into the hippocampus was different from the rest of the groups $\left(P^{\prime} \mathrm{s}<0.05\right)$, except to BLAANI group. Indicating that the hippocampal treated group showed exclusively long-term context recognition memory impairment (see Fig. 2B).

\section{Discussion}

The present study investigated the contribution of different temporal-lobe regions in the consolidation of the memory for object and object-in-context recognition tasks. These two tasks allow dissociating two important components of recognition memory, 


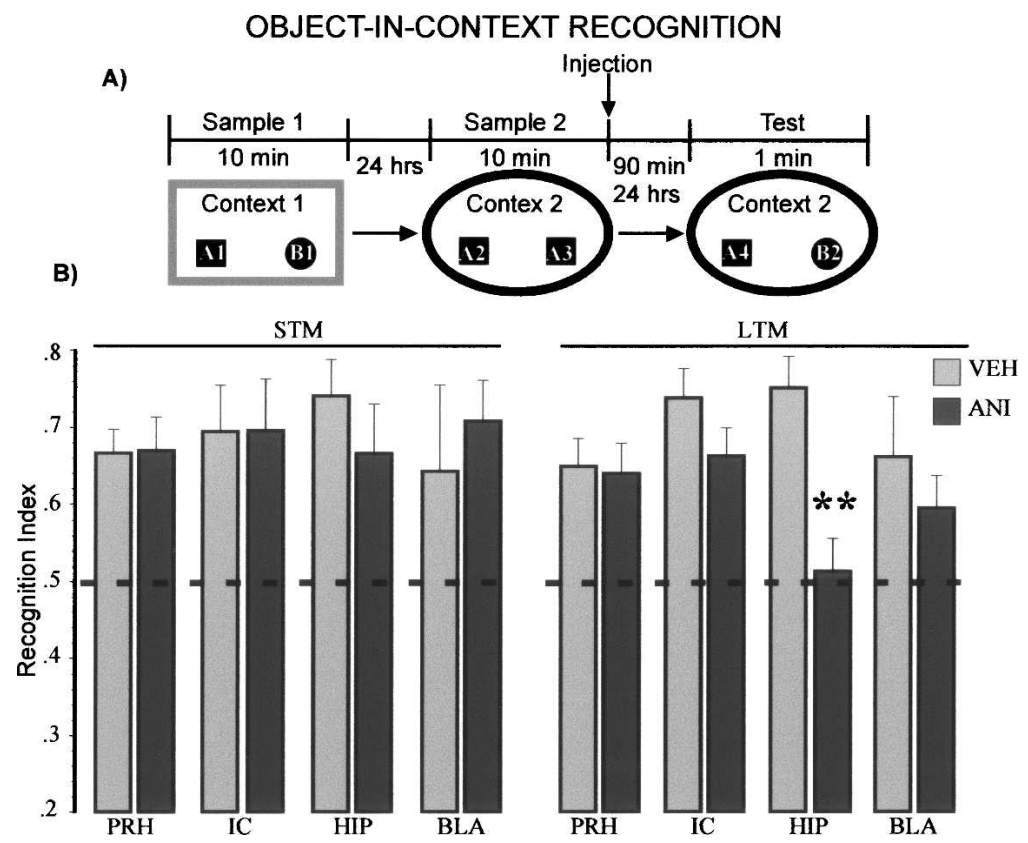

Figure 2. Protein synthesis in the hippocampus is required for long-term object-in-context recognition memory. (A) Schematic representation of the behavioral protocol used. $90 \min ($ STM), $24 \mathrm{~h}$ (LTM). (B) Recognition indexes on object-in-context recognition memory test. Two-way ANOVA for STM showed no differences between groups. Abbreviations are as in Figure 1. Two-way ANOVA for LTM showed significant differences between groups; Fisher's post hoc test revealed that the HIP-ANI group was different from the other groups $(P<0.05)$, except with the BLA-ANI group. ${ }^{* *} P<0.01$ vs. HIP-VEH.

the identity of the object (a whole representation of the stimulus), and the context where it was found. The object recognition task is based on the discrimination of familiarity of the stimuli and for normal performance the subject needs to respond to "what" stimulus was used in the experiment previously. On the other hand, the object-in-context task is based on the association of a specific stimulus with a context and for this, the subject needs to remember "where" the stimulus was first experimented. Conversely, in our object-in-context protocol either the objects or the context are familiar at the test phase; the only novel information is the relation between them.

When the protein synthesis blocker anisomycin was microinfused post-training into the perirhinal or insular cortices, object but not object-in-context recognition memory consolidation was impaired. Conversely, administration of anisomycin into the dorsal hippocampus blocked consolidation of object-in-context, but not object recognition memory. Interestingly, when anisomycin was administered into the BLA, object and object-incontext recognition memory consolidation were unaffected. In none of these regions do anisomycin infusions appear to have effects on short-term object or object-in-context recognition memory.

The ability to recognize a previously experienced stimulus has two components; recollection of the stimulus in the context where it was experienced and a sense of familiarity with the features of that particular stimulus (Eichenbaum et al. 2007). Recognition memory has been linked to a network of medial temporal cortical regions, including the perirhinal, parahippocampal, entorhinal cortices, and the hippocampus (Aggleton and Brown 1999; Malkova and Mishkin 2003; Reed et al. 2004). Recently, it was suggested that these distinct temporal lobe regions are differentially involved in object (discrimination of familiarity) and context (the place where they occur) recognition memory processes (Brown and Aggleton 2001). In this regard,
Winters and coworkers reported a functional double dissociation between the effects of hippocampus and peripostrhinal cortex lesions on object recognition and spatial memory. They found that hippocampus lesions impair spatial memory but not recognition memory and peri-postrhinal cortex lesions disrupt object recognition but not spatial memory (Winters et al. 2004). Similarly, Eacott and coworkers have demonstrated that lesions of the perirhinal cortex disrupt memory in a noncontextual object recognition task and the same lesions have no effect in an objectin-context task (Norman and Eacott 2005). However, Barker and coworkers (2007) have shown that perirhinal lesions impaired an object-in-place task that has a spatial component. In this task, the animals have to recognize that a specific object has changed position with respect to another. In their histological analysis they found that in addition to perirhinal lesions there was some damage to the lateral entorhinal cortex (Barker et al. 2007), which has been reported to disrupt spatial memory when lesioned (Bannerman et al. 2001). Altogether, our results support the doublefunctional dissociation between hippocampus and cortex and provide further evidence of the participation of these structures in recognition memory consolidation.

The perirhinal cortex is a multimodal association region that is densely interconnected with sensory areas representing many sensory modalities, allowing information exchange between the perirhinal and unimodal cortices (Burwell et al. 1995). This reciprocal connectivity, together with findings of studies that assessed perirhinal participation in recognition memory for different sensory modalities (Otto and Eichenbaum 1992; Suzuki et al. 1993; Buffalo et al. 1999; Gutierrez et al. 2004), support the idea that multiple sensory systems related to stimulus recognition activate the perirhinal cortex (Brown and Aggleton 2001; Murray and Richmond 2001). Thus, association of individual features that represent a stimulus as a whole (within-stimulus association of components) may be represented in the rhinal cortex. Complex associations between stimuli and environment (context) may be represented in the hippocampal formation (Brown and Aggleton 2001).

In support of this hypothesis, electrophysiological recording studies have reported evidence of neuronal changes related to relative familiarity of a visual stimulus in the anterior temporal lobe cortex (entorhinal and perirhinal cortices) (Baylis and Rolls 1987; Brown et al. 1987; Miller et al. 1991). Further, such responses are considerably decreased between the first and second exposure to the same item. This decremented neuronal activity persists for at least one day after stimulation and is specific to a particular item; accordingly, exposure to another novel item induces a normal response. Furthermore, the decrement in neuronal activity persists even if several other items are presented in between (Riches et al. 1991; Fahy et al. 1993; Xiang and Brown 1998). Such findings suggest that the reduced responsiveness in the entorhinal and perirhinal cortices reflects long-term memory processes in those brain regions (Brown and Xiang 1998). This decremented response may allow a better-tuned representation 
Table 2. Recognition indexes on sample phases in context recognition task

Object in-context recognition

\begin{tabular}{|c|c|c|c|c|}
\hline \multirow[b]{3}{*}{$(A)$} & \multicolumn{2}{|c|}{ Sample phase 1} & & Sample phase 2 \\
\hline & \multicolumn{4}{|c|}{ (For STM) } \\
\hline & Vehicle & Anisomycin & Vehicle & Anisomycin \\
\hline $\begin{array}{l}\text { PRH } \\
\text { IC } \\
\text { HIP } \\
\text { BLA }\end{array}$ & $\begin{array}{r}0.47 \pm 0.03 \\
0.50 \pm 0.01 \\
0.49 \pm 0.02 \\
0.55 \pm 0.02 \\
F_{(7,42)}=0 .\end{array}$ & $\begin{array}{l}0.49 \pm 0.02 \\
0.50 \pm 0.01 \\
0.49 \pm 0.01 \\
0.47 \pm 0.06 \\
0 P=0.51\end{array}$ & $\begin{array}{c}0.50 \pm 0.02 \\
0.54 \pm 0.01 \\
0.51 \pm 0.02 \\
0.52 \pm 0.02 \\
F_{(7,42)}=1\end{array}$ & $\begin{array}{l}0.47 \pm 0.02 \\
0.47 \pm 0.02 \\
0.46 \pm 0.03 \\
0.43 \pm 0.03 \\
5 P=0.12\end{array}$ \\
\hline \multirow{2}{*}{ BLA } & \multicolumn{2}{|c|}{ Sample phase 1} & \multicolumn{2}{|c|}{ Sample phase 2} \\
\hline & \multicolumn{4}{|c|}{ (For LTM) } \\
\hline (B) & Vehicle & Anisomycin & Vehicle & Anisomycin \\
\hline $\begin{array}{l}\text { PRH } \\
\text { IC } \\
\text { HIP } \\
\text { BLA }\end{array}$ & $\begin{array}{r}0.47 \pm 0.03 \\
0.47 \pm 0.02 \\
0.48 \pm 0.02 \\
0.51 \pm 0.03 \\
F_{(7,59)}=0\end{array}$ & $\begin{array}{l}0.49 \pm 0.01 \\
0.51 \pm 0.01 \\
0.53 \pm 0.02 \\
0.48 \pm 0.02 \\
1 P=0.50\end{array}$ & $\begin{array}{c}0.47 \pm 0.01 \\
0.47 \pm 0.03 \\
0.46 \pm 0.01 \\
0.49 \pm 0.01 \\
F_{(7,59)}=\end{array}$ & $\begin{array}{l}0.52 \pm 0.01 \\
0.51 \pm 0.01 \\
0.48 \pm 0.03 \\
0.51 \pm 0.03 \\
6 P=0.61\end{array}$ \\
\hline
\end{tabular}

Recognition indexes on sample phases expressed as mean \pm SEM. (A) Sample phase 1 (two different objects in context 1), and sample phase 2 (two identical objects in context 2) of STM groups. (B) Sample phase 1 (two different objects in context 1), and sample phase 2 (two identical objects in context 2) of LTM groups. Two-way ANOVA showed no differences between groups. See Results section for a detailed statistical description. Only for the purpose of analysis, one of the objects was indistinctly selected as "novel" in sample phase 2 . Abbreviations are as in Figure 1.

of a particular item (Brown and Xiang 1998; Rainer and Miller 2000; Ranganath and Rainer 2003). In contrast, no significant changes in neuronal responses were found in the hippocampus after exposure to visual stimuli (Brown et al. 1987). Considerable evidence shows that neuronal responses in the hippocampus are related to spatial components, such as self-position in space (Muller 1996). Moreover, much evidence suggests that hippocampal neuronal activity signals information concerning stimuli in particular places (Gaffan and Parker 1996).

Additional evidence of a differential involvement of cortex and hippocampus in recognition memory comes from immunohistochemical detection of the Fos protein after visual stimuli presentation. Fos protein is rapidly expressed following neuronal activation. Studies using Fos activation to detect activated individual neurons have reported that more neurons are activated by novel stimuli than by familiar items in the perirhinal cortex but not in the hippocampus. By contrast, more hippocampal and postrhinal, but not perirhinal activity was induced when a new spatial arrangement of familiar objects was presented to rats ( $\mathrm{hu}$ et al. 1995, 1996, 1997).

Pharmacological manipulations have provided additional evidence suggesting that the perirhinal and insular cortices store individual stimulus information (Bermudez-Rattoni et al. 2005; Winters and Bussey 2005a,b). Our present findings support this view and extend these previous findings by showing that memory consolidation involves the temporal lobe cortex for object, but not object-in-context, recognition memory storage.

Although particular attention has been paid to the perirhinal cortex, it is clear that other cortical regions are also important in recognition memory processing. Recently, we reported that the insular cortex is required for both object and taste recognition memory consolidation (Bermudez-Rattoni et al. 2005; Rodriguez-Ortiz et al. 2005). These results considered together with the present findings strongly indicate that temporal cortical structures located along the rhinal sulcus are critical for object recognition memory storage.

There is extensive evidence that arousal-induced activation of the amygdala regulates memory formation (McGaugh 2002, 2004). A recent paper (Canal et al. 2007) reported that anisomycin deregulates biogenic amines, including norepinephrine (NE) and dopamine (DA) when infused into the amygdala. Levels of both NE and DA are substantially increased. Thus, the memoryinfluencing effects of anisomycin infused into the BLA appear to be due, at least in part, to such deregulation. Glucocorticoids and noradrenergic receptors are activated in the BLA by emotional arousal. This synergic activity constitutes a selective neural mechanism that serves to enhance the consolidation of memories of emotional experiences (McGaugh and Roozendaal 2002; Roozendaal et al. 2006). Importantly, this modulatory system does not seem to occur in other regions, for example, the hippocampus (Roozendaal et al. 2006). In the present experiments anisomycin microinfused into the BLA post-training did not affect either object or object-in-context recognition memory consolidation. This finding was unexpected in view of evidence that post-training BLA activation induced by microinfusions of the muscarinic cholinergic agonist oxotremorine enhances spatial memory (Malin and McGaugh 2006). Training that induces poor retention is typically used to investigate memory modulation induced by BLA activation. In the present study we obtained high recognition memory scores and, thus, the experimental conditions used may not be suitable to address the participation of modulatory systems.

Other studies have evaluated the role of the amygdala in information processing by means of object novelty detection and novel stimuli relationships. Moses and coworkers (Moses et al. 2005) found that rats with neurotoxic lesions of the amygdala showed disrupted responses to novel objects and object relationships. However, they reported extra-amygdalar damage to adjacent structures including the ventral bank of the perirhinal cortex, making it difficult to conclude the specific participation of the amygdala in recognition memory processing.

Importantly, our findings clearly indicate that the hippocampus is required for object-in-context recognition memory consolidation. However, studies using permanent lesions have reported that the hippocampus is required to enable animals to remember familiar objects in novel locations for a short period of time (5 min) (Mumby et al. 2002). The results reported in the present study contribute to two important additional features. First with a longer training, retention can be assessed at least 24 $\mathrm{h}$ later, allowing long-term evaluation of object-context association memory. Second, most of the cerebral manipulations used in previous studies made it difficult to determine the involvement of a particular structure in a specific process of memory formation. Under our protocol it was possible to specifically assess their particular roles in object and object-in-context memory consolidation separately.

However, a recent paper reported evidence suggesting that protein synthesis in the hippocampus is necessary for consolidation of object recognition memory (Rossato et al. 2007). Contrary to the protocol used in the present experiment, two different objects were presented in the sample phase of the object recognition task and on the test; however, the position of the familiar objects was interchanged. This protocol may provide important contextual information to memory and might explain hippocampus participation. Consistently, Mumby and coworkers reported that lesions of the hippocampus disrupt recognition memory only when relative positions of the objects are changed (Mumby et al. 2002).

With the protocols described here it is possible to differen- 
tially assess the two components of recognition memory for short- and long-term memory. Additionally, particular memory phases can be evaluated separately. Our findings provide additional information concerning the participation of distinct structures of the temporal lobe required for recognition memory processing, thus making it clear that the hippocampus and the cortex have specific and different roles in long-lasting recognition memory storage.

\section{Materials and Methods}

\section{Subjects}

Subjects were adult male Wistar rats weighing between 280 and $300 \mathrm{~g}$ at the beginning of the experiment. Animals were obtained from the breeding colony of the Institute of Cellular Physiology, UNAM. They were maintained at room temperature $\left(22^{\circ} \mathrm{C}-23^{\circ} \mathrm{C}\right)$, under a 12-12 h light-dark schedule, caged individually in a standard acrylic box with food and water freely available. All behavioral protocols were carried out during the light phase. All experiments were performed in accordance with the Ministry of Health, Mexico.

\section{Surgery and microinfusions}

Before surgery rats were deeply anesthetized with intraperitonial injections of ketamine $(83.49 \mathrm{mg} / \mathrm{kg})$ and xylazine $(8.58 \mathrm{mg} / \mathrm{kg})$. Rats were implanted bilaterally with guide cannulae aimed either at the perirhinal cortex (PRH), (coordinates of the infusion sites from bregma [mm] [Paxinos and Watson 1986]): posterior 3, lateral \pm 6.5 , ventral 7 ; insular cortex (IC): anterior 1.2 , lateral \pm 5.5 , ventral 6.5; hippocampus (HIP): posterior 3.6, lateral \pm 3.0, ventral 3.3; and basolateral amygdala (BLA): posterior 2.8 , lateral \pm 5 , ventral 8.5. Cannulae were anchored with dental acrylic cement to two surgical screws fixed to the skull. Stylets were inserted into guide cannulae to prevent clogging. Animals were allowed to recuperate for $1 \mathrm{wk}$ before being subjected to behavioral procedures.

For microinfusions, stylets were removed and a 30-gauge infusion needle was inserted extending $2.0 \mathrm{~mm}$ from the tip of each guide cannula. The infusion needle was connected via polyethylene tubing to a $10 \mu \mathrm{L}$ Hamilton microsyringe driven by a microinfusion pump (Cole Parmer Instruments). In all cases, injections were performed over a minute and the injector was left in place for an additional minute to allow complete diffusion. For IC, PRH, and HIP the injected volume was $1 \mu \mathrm{L}$ per hemisphere; for BLA the injected volume was $0.5 \mu \mathrm{L}$ per hemisphere. Microinjections of vehicle solution served as the control.

\section{Drugs}

The protein synthesis inhibitor anisomycin was purchased from Sigma (St. Louis). The drug was dissolved in equimolar $\mathrm{HCl}$ and adjusted to $120 \mathrm{mg} / \mathrm{mL}, \mathrm{pH} \sim 7.4$ in vehicle solution (ACSF [mM]: $125 \mathrm{NaCl}, 5 \mathrm{KCl}, 1.25 \mathrm{NaH}_{2} \mathrm{PO}_{4} \mathrm{H}_{2} \mathrm{O}, 1.5 \mathrm{MgSO}_{4} 7 \mathrm{H}_{2} \mathrm{O}, 26$ $\mathrm{NaHCO}_{3}, 10$ glucose, $2.5 \mathrm{CaCl}_{2}$ ). The dose of anisomycin was chosen according to previous studies indicating that doses between 100 and $125 \mu \mathrm{g} / \mu \mathrm{L}$ were effective in inhibiting protein synthesis in more than $90 \%$ indistinctly of the infused structure (Rosenblum et al. 1993; Morris et al. 2006; Canal et al. 2007) the volume was chosen according to a previous study that shows that $1 \mu \mathrm{L}$ of ink into the cortex spreads $1 \mathrm{~mm}$ on average (Berman et al. 2000).

\section{Apparatus}

On object recognition memory experiments, one square arena made of graypainted wood with the floor covered with sawdust was used $(40 \times 40 \times 40$ $\mathrm{cm})$. On object-in-context recognition memory experiments, we used two arenas with different physical features. A similar gray square arena served as one of the contexts, and a circular arena made of red plastic material (40 cm in height $\times 40 \mathrm{~cm}$ in diameter) was used as the other context. For both arenas, some irregular figures made of cardboard were placed in the walls to serve as spatial landmarks, and the floor was covered with sawdust.

The arenas were placed in the same dim-light illuminated room. A video camera was mounted above the open-field arenas and all test sessions were recorded. The objects to be discriminated were white glass bulbs $(6 \mathrm{~cm}$ in diameter and $11 \mathrm{~cm}$ in length) and transparent glass jars $(5.5 \mathrm{~cm}$ in diameter and $5 \mathrm{~cm}$ in height). All objects were fixed to the floor at the back corners of the arena ( $10 \mathrm{~cm}$ from walls) with Velcro to prevent them from being displaced by the rats. To avoid olfactory cues, objects were thoroughly cleaned with $70 \%$ ethanol and the sawdust was stirred after each trial.

\section{Behavioral procedures}

For each session, animals were transported from the vivarium to the experimental room $2 \mathrm{~h}$ before the beginning of each session, and were left in the experimental room for an additional $2 \mathrm{~h}$ at the end of each session, in order to avoid stress conditions that could affect the performance or consolidation of the tasks.

For all experiments, contexts, objects and their relative positions were counterbalanced. All experiments were carried out in independent groups. Exploration was considered as pointing the nose toward an object at a distance of less than $1 \mathrm{~cm}$ and/or touching it with the nose. Turning around or sitting on the objects was not considered as exploratory behavior. Rats that showed a total exploration time $<10 \mathrm{~s}$ on either training or testing were discarded from further analysis.

\section{Object recognition memory task}

For five consecutive days animals were handled for a minute, and immediately after positioned into the open-field arena without any objects for $3 \mathrm{~min}$. On the sample phase, rats were placed in the arena facing the wall opposite the objects for $10 \mathrm{~min}$ and were allowed to freely explore two identical objects (either two light bulbs or two jars $\left[A_{1}\right.$ and $\left.A_{2}\right]$ ). Memory was tested either 90 min (short-term memory) or $24 \mathrm{~h}$ later (long-term memory). On memory test, rats were allowed to explore freely one copy of the previously presented object (familiar, $\mathrm{A}_{3}$ ) together with a new one (B) for $3 \mathrm{~min}$. Vehicle or anisomycin was microinjected immediately after the sample phase (see Fig. 1A). The groups tested for STM were: PRH-VEH $(n=4)$, IC-VEH $(n=6)$, HIP-VEH $(n=8)$, BLA-VEH $(n=7)$; PRH-ANI $(n=7)$, IC-ANI $(n=5)$, HIP-ANI $(n=5)$, BLA-ANI $(n=8)$. The groups used for LTM were: PRH-VEH $(\mathrm{n}=9)$, IC-VEH $(\mathrm{n}=11)$, HIP-VEH $(\mathrm{n}=9)$, BLA-VEH $(n=8)$; PRHANI $(n=10)$, IC-ANI $(n=11)$, HIP-ANI $(n=12)$, BLA-ANI $(n=6)$. The object recognition index was calculated as follows: time of exploration of novel object/(time of exploration of familiar object + time of exploration of novel object) (Ennaceur and Delacour 1988). A recognition index equal to 0.5 reflects no preference for any of the objects. An index higher than 0.5 shows preferences for novel objects.

\section{Object-in-context recognition memory task}

For five consecutive days animals were handled for $1 \mathrm{~min}$, and immediately after habituated to both contexts (90 min apart) without any objects for $3 \mathrm{~min}$. On sample phase 1 , rats were placed in one of the arenas (context 1 ) facing the wall opposite to
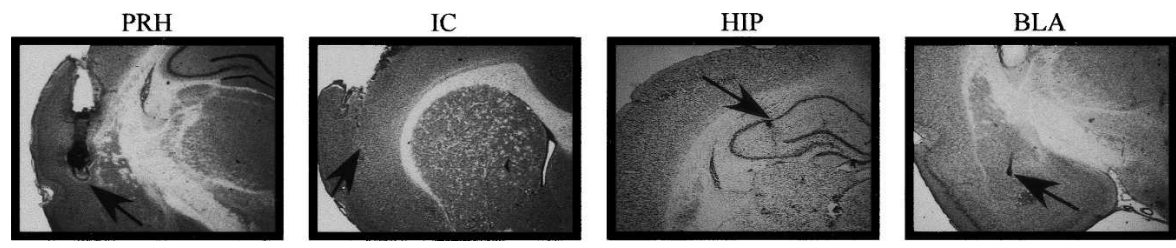

Figure 3. Representative photographs of infusion sites, the arrows point to the needle tips; abbreviations as in Figure 1. 
the objects and were allowed to explore two different objects (one light bulb and one jar $\left[\mathrm{A}_{1}\right.$ and $\left.\mathrm{B}_{1}\right]$ ) for $10 \mathrm{~min}$. Sample phase 2 was conducted $24 \mathrm{~h}$ later, rats were placed in context 2 together with two identical objects (copies of one of the previously presented objects, i.e., either two light bulbs or two jars $\left[A_{2}\right.$ and $\left.A_{3}\right]$ ) and were allowed to explore the objects for $10 \mathrm{~min}$. Memory was tested $90 \mathrm{~min}$ (short-term memory) or $24 \mathrm{~h}$ (long-term memory) later. On the memory test, rats were reintroduced to context 2 and were allowed to explore freely for $3 \mathrm{~min}$ one copy of the previously presented object in context $2\left(\mathrm{~A}_{4}\right)$ together with a copy of one of the objects previously presented in context 1 but not presented in context $2\left(\mathrm{~B}_{2}\right)$. Vehicle or anisomycin was microinjected immediately after sample phase 2 in order to assess consolidation of familiar objects presented in a novel context (see Fig. 2A). The groups tested for STM were: PRH-VEH $(n=7)$, IC-VEH $(n=5)$, HIP-VEH $(n=7)$, BLA-VEH $(n=7)$; PRH-ANI $(n=7)$, IC-ANI $(n=5)$, HIP-ANI $(n=7)$, BLA-ANI $(n=5)$. For LTM the groups used were: PRH-VEH $(n=10)$, IC-VEH $(n=6)$, HIPVEH $(n=6)$, BLA-VEH $(n=8)$; PRH-ANI $(n=11)$, IC-ANI $(n=9)$, HIP-ANI $(n=8)$, BLA-ANI $(n=9)$. Context recognition indexes were calculated as follows: time of exploration of familiar object in novel context/(time of exploration of familiar object in familiar context + time of exploration of familiar object in novel context). A recognition index equal to 0.5 reflects no preference for any of the objects. An index higher than 0.5 shows a preference for familiar objects in novel contexts.

In the protocol designed for object-in-context recognition memory assessment, one of the objects was presented twice before the retention test, while the other was presented just once, raising the possibility of one of the objects becoming more familiar. To discard the possibility that one object may become more familiar than the other on the retention test, we conducted the following control experiment.

On sample phase 1 , rats were placed in one of the arenas (context 1) facing the wall opposite the objects and allowing them to explore two different objects (a light bulb and a jar $\left[\mathrm{A}_{1}\right.$ and $\left.B_{1}\right]$ ) for $10 \mathrm{~min}$. Sample phase 2 was conducted $24 \mathrm{~h}$ later, rats were placed in context 2 with two copies of one of the familiar objects (either two light bulbs or two jars $\left[\mathrm{A}_{2}\right.$ and $\left.\mathrm{A}_{3}\right]$ ) and were allowed to explore the objects for $10 \mathrm{~min}$. Retention was tested $24 \mathrm{~h}$ after sample phase 2 (long-term memory test). During the retention test, rats were reintroduced to context 1 and were allowed to explore freely for $3 \mathrm{~min}$ one copy of each of the previously presented objects $\left(A_{4} B_{2}\right)$.

Under these conditions, we expected that rats would explore equally the two objects either in sample phases or in memory test, because there are not any novelty components for discrimination. One-sample $t$-test showed that the sample phases and memory test were not different from the 0.5 score (chance level) $P>0.05$ ( $n=14$, data not shown).

\section{Histology}

At the end of the experiments, animals were overdosed with sodium pentobarbital, and perfused with $0.9 \%$ saline. The brains were removed and stored at $4{ }^{\circ} \mathrm{C}$ in buffered $4 \%$ paraformaldehyde solution for $24 \mathrm{~h}$. The brains were then stored in a $30 \%$ sucrose/phosphate buffer $(0.1 \mathrm{M})$ solution for five days. Frozen sections $(40 \mu \mathrm{m})$ were collected and stained with Cresyl violet and examined by light microscopy in order to establish injector tips placement. All animals included in the analysis had the needle tips in the cerebral region of interest. Figure 3 shows representative microphotographs of the injection placements.

\section{Statistical analysis}

Although the memory test was recorded for a total time of $3 \mathrm{~min}$, for the context recognition task only the first minute was used for statistical analysis, since it has been reported that novel object discrimination is more evident during the first minute (Dix and Aggleton 1999; Mumby et al. 2002). The analysis for the object recognition task included the whole $3 \mathrm{~min}$. Two-way ANOVA (drug $\times$ brain area) was used to assess behavioral data. Mean \pm SEM recognition indexes were used for comparisons be- tween groups. A probability level of $P<0.05$ was accepted as statistical significance.

\section{Acknowledgments}

This research was supported by CONACYT-México 60478 and DGAPA-UNAM IN220706-3 to F.B.-R., and NIMH 12526 to J.L.M. We acknowledge Azul Islas for assisting in the experiments and Oreste Carbajal for technical support.

\section{References}

Aggleton, J.P. and Brown, M.W. 1999. Episodic memory, amnesia, and the hippocampal-anterior thalamic axis. Behav. Brain Sci. 22: 425-444; discussion 444-489.

Bannerman, D.M., Yee, B.K., Lemaire, M., Wilbrecht, L., Jarrard, L., Iversen, S.D., Rawlins, J.N., and Good, M.A. 2001. The role of the entorhinal cortex in two forms of spatial learning and memory. Exp. Brain Res. 141: 281-303.

Barker, G.R., Bird, F., Alexander, V., and Warburton, E.C. 2007. Recognition memory for objects, place, and temporal order: A disconnection analysis of the role of the medial prefrontal cortex and perirhinal cortex. J. Neurosci. 27: 2948-2957.

Baylis, G.C. and Rolls, E.T. 1987. Responses of neurons in the inferior temporal cortex in short term and serial recognition memory tasks. Exp. Brain Res. 65: 614-622.

Berman, D.E., Hazvi, S., Neduva, V., and Dudai, Y. 2000. The role of identified neurotransmitter systems in the response of insular cortex to unfamiliar taste: Activation of ERK1-2 and formation of a memory trace. J. Neurosci. 20: 7017-7023.

Bermudez-Rattoni, F. 2004. Molecular mechanims of taste-recognition memory. Nat. Rev. Neurosci. 5: 209-217.

Bermudez-Rattoni, F., Okuda, S., Roozendaal, B., and McGaugh, J.L. 2005. Insular cortex is involved in consolidation of object recognition memory. Learn. Mem. 12: 447-449.

Broadbent, N.J., Squire, L.R., and Clark, R.E. 2004. Spatial memory, recognition memory, and the hippocampus. Proc. Natl. Acad. Sci. 101: $14515-14520$.

Brown, M.W. and Aggleton, J.P. 2001. Recognition memory: What are the roles of the perirhinal cortex and hippocampus? Nat. Rev. Neurosci. 2: 51-61.

Brown, M.W. and Xiang, J.Z. 1998. Recognition memory: Neuronal substrates of the judgement of prior occurrence. Prog. Neurobiol. 55: $149-189$.

Brown, M.W., Wilson, F.A., and Riches, I.P. 1987. Neuronal evidence that inferomedial temporal cortex is more important than hippocampus in certain processes underlying recognition memory. Brain Res. 409: 158-162.

Buffalo, E.A., Ramus, S.J., Clark, R.E., Teng, E., Squire, L.R., and Zola, S.M. 1999. Dissociation between the effects of damage to perirhinal cortex and area TE. Learn. Mem. 6: 572-599.

Burwell, R.D., Witter, M.P., and Amaral, D.G. 1995. Perirhinal and postrhinal cortices of the rat: A review of the neuroanatomical literature and comparison with findings from the monkey brain. Hippocampus 5: 390-408.

Bussey, T.J., Muir, J.L., and Aggleton, J.P. 1999. Functionally dissociating aspects of event memory: The effects of combined perirhinal and postrhinal cortex lesions on object and place memory in the rat. $J$. Neurosci. 19: 495-502.

Canal, C.E., Chang, Q., and Gold, P.E. 2007. Amnesia produced by altered release of neurotransmitters after intraamygdala injections of a protein synthesis inhibitor. Proc. Natl. Acad. Sci. 104: $12500-12505$.

Dix, S.L. and Aggleton, J.P. 1999. Extending the spontaneous preference test of recognition: Evidence of object-location and object-context recognition. Behav. Brain Res. 99: 191-200.

Eichenbaum, H., Yonelinas, A.P., and Ranganath, C. 2007. The medial temporal lobe and recognition memory. Annu. Rev. Neurosci. 30: $123-152$.

Ennaceur, A. and Aggleton, J.P. 1997. The effects of neurotoxic lesions of the perirhinal cortex combined to fornix transection on object recognition memory in the rat. Behav. Brain Res. 88: 181-193.

Ennaceur, A. and Delacour, J. 1988. A new one-trial test for neurobiological studies of memory in rats. 1: Behavioral data. Behav. Brain Res. 31: 47-59.

Ennaceur, A., Neave, N., and Aggleton, J.P. 1996. Neurotoxic lesions of the perirhinal cortex do not mimic the behavioural effects of fornix transection in the rat. Behav. Brain Res. 80: 9-25.

Fahy, F.L., Riches, I.P., and Brown, M.W. 1993. Neuronal activity related to visual recognition memory: Long-term memory and the encoding of recency and familiarity information in the primate anterior and 
medial inferior temporal and rhinal cortex. Exp. Brain Res. 96: $457-472$.

Gaffan, D. and Parker, A. 1996. Interaction of perirhinal cortex with the fornix-fimbria: Memory for objects and "object-in-place" memory. J. Neurosci. 16: 5864-5869.

Gutierrez, R., De la Cruz, V., Rodriguez-Ortiz, C.J., and Bermudez-Rattoni, F. 2004. Perirhinal cortex muscarinic receptor blockade impairs taste recognition memory formation. Learn. Mem. 11: $95-101$.

Malin, E.L. and McGaugh, J.L. 2006. Differential involvement of the hippocampus, anterior cingulate cortex, and basolateral amygdala in memory for context and footshock. Proc. Natl. Acad. Sci. 103: $1959-1963$.

Malkova, L. and Mishkin, M. 2003. One-trial memory for object-place associations after separate lesions of hippocampus and posterior parahippocampal region in the monkey. J. Neurosci. 23: 1956-1965

Mandler, G. 1980. The judgment of previous occurence. Psychol. Rev. 87: $252-271$.

McGaugh, J.L. 2002. Memory consolidation and the amygdala: A systems perspective. Trends Neurosci. 25: 456 . doi: 10.1016/S0166-2236(02)02211-7.

McGaugh, J.L. 2004. The amygdala modulates the consolidation of memories of emotionally arousing experiences. Annu. Rev. Neurosci. 27: $1-28$.

McGaugh, J.L. and Roozendaal, B. 2002. Role of adrenal stress hormones in forming lasting memories in the brain. Curr. Opin. Neurobiol. 12: $205-210$.

Miller, E.K., Li, L., and Desimone, R. 1991. A neural mechanism for working and recognition memory in inferior temporal cortex. Science 254: $1377-1379$.

Mishkin, M. 1978. Memory in monkeys severely impaired by combined but not by separate removal of amygdala and hippocampus. Nature 273: $297-298$

Morris, R.G., Inglis, J., Ainge, J.A., Olverman, H.J., Tulloch, J., Dudai, Y., and Kelly, P.A. 2006. Memory reconsolidation: Sensitivity of spatial memory to inhibition of protein synthesis in dorsal hippocampus during encoding and retrieval. Neuron 50: 479-489.

Moses, S.N., Cole, C., Driscoll, I., and Ryan, J.D. 2005. Differential contributions of hippocampus, amygdala and perirhinal cortex to recognition of novel objects, contextual stimuli and stimulus relationships. Brain Res. Bull. 67: 62-76.

Muller, R. 1996. A quarter of a century of place cells. Neuron 17: 813-822

Mumby, D.G., Gaskin, S., Glenn, M.J., Schramek, T.E., and Lehmann, H. 2002. Hippocampal damage and exploratory preferences in rats: Memory for objects, places, and contexts. Learn. Mem. 9: 49-57.

Mumby, D.G., Tremblay, A., Lecluse, V., and Lehmann, H. 2005. Hippocampal damage and anterograde object-recognition in rats after long retention intervals. Hippocampus 15: 1050-1056.

Murray, E.A. and Mishkin, M. 1998. Object recognition and location memory in monkeys with excitotoxic lesions of the amygdala and hippocampus. J. Neurosci. 18: 6568-6582.

Murray, E.A. and Richmond, B.J. 2001. Role of perirhinal cortex in object perception, memory, and associations. Curr. Opin. Neurobiol. 11: $188-193$

Norman, G. and Eacott, M.J. 2005. Dissociable effects of lesions to the perirhinal cortex and the postrhinal cortex on memory for context and objects in rats. Behav. Neurosci. 119: 557-566.

Otto, T. and Eichenbaum, H. 1992. Complementary roles of the orbital prefrontal cortex and the perirhinal-entorhinal cortices in an odor-guided delayed-nonmatching-to-sample task. Behav. Neurosci. 106: $762-775$.

Paxinos, G. and Watson, C. 1986. The rat brain in stereotaxic coordinates (3rd ed.). Academic Press, San Diego, CA.

Rainer, G. and Miller, E.K. 2000. Effects of visual experience on the representation of objects in the prefrontal cortex. Neuron 27: $179-189$.

Ranganath, C. and Rainer, G. 2003. Neural mechanisms for detecting and remembering novel events. Nat. Rev. Neurosci. 4: 193-202.

Reed, C.L., Shoham, S., and Halgren, E. 2004. Neural substrates of tactile object recognition: An fMRI study. Hum. Brain Mapp. 21: 236-246.

Riches, I.P., Wilson, F.A., and Brown, M.W. 1991. The effects of visual stimulation and memory on neurons of the hippocampal formation and the neighboring parahippocampal gyrus and inferior temporal cortex of the primate. J. Neurosci. 11: 1763-1779.

Rodriguez-Ortiz, C.J., De la Cruz, V., Gutierrez, R., and Bermudez-Rattoni, F. 2005. Protein synthesis underlies post-retrieval memory consolidation to a restricted degree only when updated information is obtained. Learn. Mem. 12: 533-537.

Roozendaal, B., Okuda, S., Van der Zee, E.A., and McGaugh, J.L. 2006. Glucocorticoid enhancement of memory requires arousal-induced noradrenergic activation in the basolateral amygdala. Proc. Natl. Acad. Sci. 103: 6741-6746.

Rosenblum, K., Meiri, N., and Dudai, Y. 1993. Taste memory: The role of protein synthesis in gustatory cortex. Behav. Neural Biol. 59: $49-56$.

Rossato, J.I., Bevilaqua, L.R., Myskiw, J.C., Medina, J.H., Izquierdo, I., and Cammarota, M. 2007. On the role of hippocampal protein synthesis in the consolidation and reconsolidation of object recognition memory. Learn. Mem. 14: 36-46.

Scoville, W.B. and Milner, B. 1957. Loss of recent memory after bilateral hippocampal lesions. J. Neurol. Neurosurg. Psychiatr. 20: 11-21.

Squire, L.R., Wixted, J.T., and Clark, R.E. 2007. Recognition memory and the medial temporal lobe: A new perspective. Nat. Rev. Neurosci. 8: $872-883$.

Stupien, G., Florian, C., and Roullet, P. 2003. Involvement of the hippocampal CA3-region in acquisition and in memory consolidation of spatial but not in object information in mice. Neurobiol. Learn. Mem. 80: 32-41.

Suzuki, W.A., Zola-Morgan, S., Squire, L.R., and Amaral, D.G. 1993. Lesions of the perirhinal and parahippocampal cortices in the monkey produce long-lasting memory impairment in the visual and tactual modalities. I. Neurosci. 13: 2430-2451.

Winters, B.D. and Bussey, T.J. 2005a. Glutamate receptors in perirhinal cortex mediate encoding, retrieval, and consolidation of object recognition memory. J. Neurosci. 25: 4243-4251.

Winters, B.D. and Bussey, T.J. 2005b. Transient inactivation of perirhinal cortex disrupts encoding, retrieval, and consolidation of object recognition memory. J. Neurosci. 25: 52-61.

Winters, B.D., Forwood, S.E., Cowell, R.A., Saksida, L.M., and Bussey, T.J. 2004. Double dissociation between the effects of peri-postrhinal cortex and hippocampal lesions on tests of object recognition and spatial memory: Heterogeneity of function within the temporal lobe. J. Neurosci. 24: 5901-5908.

Xiang, J.Z. and Brown, M.W. 1998. Differential neuronal encoding of novelty, familiarity and recency in regions of the anterior temporal lobe. Neuropharmacology 37: 657-676.

Yonelinas, A.P., Kroll, N.E., Quamme, J.R., Lazzara, M.M., Sauve, M.J., Widaman, K.F., and Knight, R.T. 2002. Effects of extensive temporal lobe damage or mild hypoxia on recollection and familiarity. Nat. Neurosci. 5: 1236-1241.

Zhu, X.O., Brown, M.W., McCabe, B.J., and Aggleton, J.P. 1995. Effects of the novelty or familiarity of visual stimuli on the expression of the immediate early gene c-fos in rat brain. Neuroscience 69: 821-829.

Zhu, X.O., McCabe, B.J., Aggleton, J.P., and Brown, M.W. 1996 Mapping visual recognition memory through expression of the immediate early gene c-fos. Neuroreport 7: 1871-1875.

Zhu, X.O., McCabe, B.J., Aggleton, J.P., and Brown, M.W. 1997. Differential activation of the rat hippocampus and perirhinal cortex by novel visual stimuli and a novel environment. Neurosci. Lett. 229: $141-143$.

Received April 14, 2008; accepted in revised form June 19, 2008. 


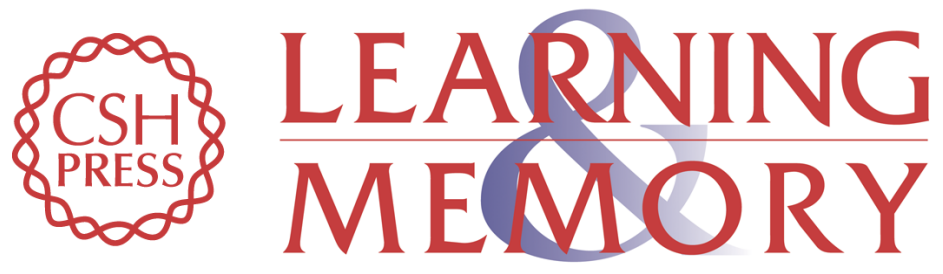

\section{The consolidation of object and context recognition memory involve different regions of the temporal lobe}

Israela Balderas, Carlos J. Rodriguez-Ortiz, Paloma Salgado-Tonda, et al.

Learn. Mem. 2008, 15:

Access the most recent version at doi:10.1101//m.1028008

References This article cites 60 articles, 23 of which can be accessed free at:

http://learnmem.cshlp.org/content/15/9/618.full.html\#ref-list-1

License

Email Alerting Receive free email alerts when new articles cite this article - sign up in the box at the Service top right corner of the article or click here. 